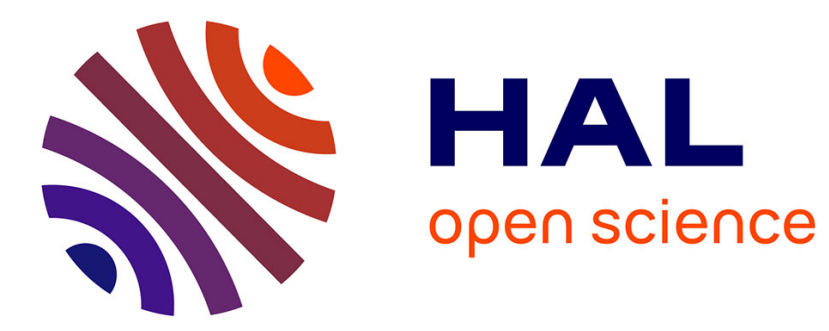

\title{
Avenir d'une identité industrielle et gestion du ou des risque(s) : Pierre-Bénite (69) dans le couloir de la chimie Violaine Girard
}

\section{To cite this version:}

Violaine Girard. Avenir d'une identité industrielle et gestion du ou des risque(s): Pierre-Bénite (69) dans le couloir de la chimie. Les Annales de la Recherche Urbaine, 2004, 95, pp.27-33. hal-02373999

\section{HAL Id: hal-02373999}

\section{https://hal-normandie-univ.archives-ouvertes.fr/hal-02373999}

Submitted on 21 Nov 2019

HAL is a multi-disciplinary open access archive for the deposit and dissemination of scientific research documents, whether they are published or not. The documents may come from teaching and research institutions in France or abroad, or from public or private research centers.
L'archive ouverte pluridisciplinaire HAL, est destinée au dépôt et à la diffusion de documents scientifiques de niveau recherche, publiés ou non, émanant des établissements d'enseignement et de recherche français ou étrangers, des laboratoires publics ou privés. 


\section{Violaine Girard}

\section{AVENIR D'UNE IDENTITÉ INDUSTRIELLE ET GESTION DU OU DES RISQUE(S) :}

PIERRE-BÉNITE (69) DANS LE COULOIR DE LA CHIMIE

$\mathbf{L}^{\mathrm{c}}$ e risque technologique peut être considéré comme une représentation sociale, fondée sur l'anticipation de dangers liés à l'activité industrielle par un groupe social mobilisant ses savoirs, son expérience et ses activités afin de s'en prémunirr. On peut alors se demander si les modalités de gestion du risque, et les réactions qu'elles suscitent localement, ne font pas intervenir les rapports autant symboliques que pratiques établis dans la longue durée entre un site de production industrielle et une société locale : comment y sont envisagés le rôle et la place de l'industrie? Quelles sont alors les positions et les actions engagées pour se prémunir de l'exposition à des accidents potentiels? Face aux dispositifs de prévention, comment les pouvoirs publics locaux construisent-ils l'avenir et élaborent-ils leurs projets urbains ?

Pour répondre à ce type de questionnement, une démarche attentive aux dimensions historiques, sociales et spatiales du territoire s'avère nécessaire. La recherche a été menée sur la commune de PierreBénite, située dans le «Couloir de la chimie», au sud de Lyon ${ }^{2}$. Y est implanté depuis près d'un siècle un site de production chimique actuellement géré par le groupe Total-Fina-Elf. Cette commune est fortement contrainte en termes de développement par l'absence d'espaces libres et la densité de l'habitat ; elle est dirigée depuis I97I par des municipalités affiliées au PCF, avec un maire ayant effectué trente ans de mandats, avant que sa première adjointe ne lui succède en 200 I. Les élus, chargés de promouvoir les intérêts communaux, sont par ailleurs responsables de la gestion urbaine du risque technologique depuis un peu plus d'une dizaine d'années's.

\section{L'usine chimique, moteur de l'histoire locale ?}

La commune de Pierre-Bénite, auparavant hameau d'Oullins, accède à l'indépendance au début du siècle grâce à l'accroissement de population lié au phénomène massif d'industrialisation de la banlieue sud de Lyon4. Par la suite, l'essor de la commune s'est pour- suivi sous l'impulsion du développement du site de la société rhône-alpine d'Électro-Chimie, implantée en I9I7. Devenu au cours de la première moitié du siècle un pôle industriel majeur, ce site a conditionné la structuration économique et morphologique de la commune, et a amené de nombreux migrants, contribuant ainsi à la croissance soutenue de la population, qui passe de 3000 habitants en I900 à 5300 en I93I. Mais Pierre-Bénite ayant connu une industrialisation plus tardive que certaines communes de la banlieue lyonnaise, ne présente pas dans cette première moitié du siècle des caractéristiques ouvrières aussi nettes, notamment en ce qui concerne l'ancrage politique du pouvoir municipal.

À partir des années i960, des phénomènes de grande ampleur touchent la commune. Le mouvement de concentration de l'industrie chimique induit la succession, à la tête du site, de plusieurs grands

I. Voir l'article de Thierry Coanus, François Duchêne et Emmanuel Martinais, dans ce numéro.

2. L'enquête de terrain s'est déroulée de novembre 200I à août 2002, soit deux mois à peine après l'explosion de l'usine AZF à Toulouse, par entretiens auprès de responsables politiques ayant participé aux différentes municipalités communistes, et auprès des aménageurs intervenant sur le territoire pierre-bénitain. Nous avons également constitué un corpus de documents produits par les municipalités, en particulier les projets urbains. La presse locale (I974-2002) a constitué une source complémentaire.

3. Ils sont en effet chargés de l'application des procédures de maîtrise de l'urbanisation, selon la directive européenne SEVESO I de I982 et la loi du 22 juillet I987. Ces textes ont instauré l'obligation pour les élus locaux d'inscrire dans les documents d'urbanisme un zonage des risques, afin d'éviter la densification autour des installations industrielles dangereuses.

4. Sur cette période de l'histoire de Pierre-Bénite, $c f$. Lequin Y., (I974), Les ouvriers de la région lyonnaise (1848-1914). La formation de la classe ouvrière régionale, Presses Universitaires de Lyon; Laferrère M., (1977), Lyon ville industrielle : essai d'une géographie urbaine des techniques et des entreprises, Paris, Presses Universitaires de France; Pitiot L., (I978), Pierre-Bénite sur Rhône, Éd. Saint-Martin-en-Haut, A. et J.-P. Martinière.

Les Annales de la recherche urbaine n 95, 0180-930-VI-04/95/pp. 27-33 @ METATTM. 
groupes 5 , plus lointains que ne l'était l'Électro-Chimie. Ces groupes mettent en place une chimie de gros tonnage, et procèdent à des restructurations se traduisant notamment par une chute continuelle du nombre d'ouvriers, après la période précédente de hausse. Dans le même temps, la ville est le siège de grands travaux d'intérêt supracommunal, promus par l'État : un barrage hydro-électrique sur le Rhône et l'autoroute A7, qui modifient considérablement le territoire communal en viabilisant les terrains constituant les berges jusqu'alors sauvages du Rhône. Puis deux projets d'agglomération sont réalisés, dans un contexte de poussée démographique et de développement de la région lyonnaise des années i960 et I970: la déviation du centre-ville par le boulevard de l'Europe et la construction d'immeubles collectifs à Haute-Roche (cf. la carte urbanisation et infrastructures). Cet ensemble d'habitat social contribue alors certainement à asseoir dans la commune une population que l'on peut qualifier de modeste ou de populaire, alors que la ville connaît la croissance la plus forte de son histoire, pour atteindre Io ooo habitants en I975.

Si l'influence du site de production chimique a été déterminante, la commune n'a pas connu pour autant

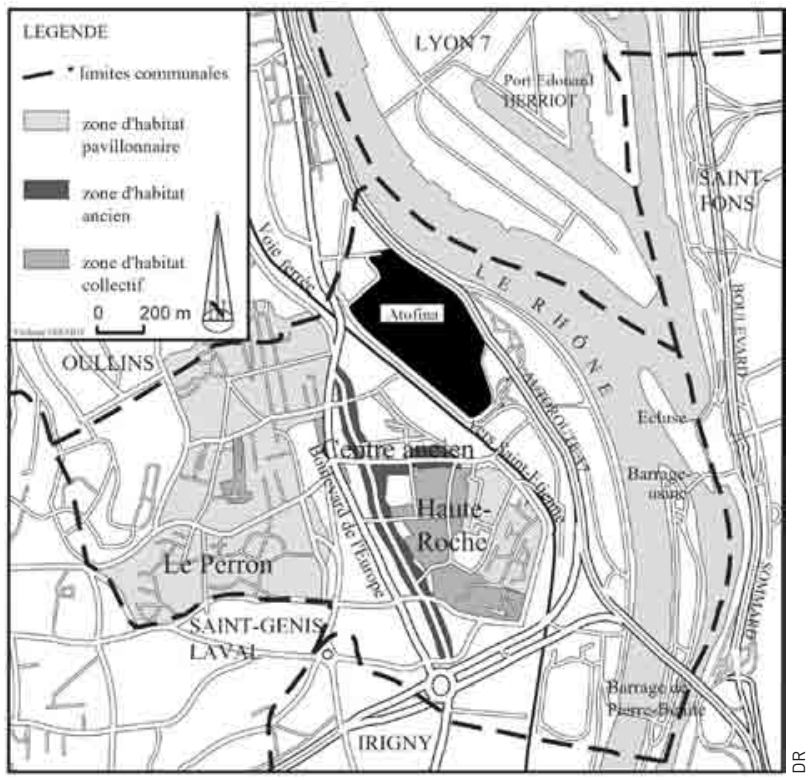

La commune de Pierre-Bénite : infrastructures et urbanisation.

une structuration mono-industrielle. Du fait de son intégration à la banlieue lyonnaise, la ville a rencontré tout au long de son histoire l'influence de facteurs multiples liés à la dynamique de l'agglomération. Dans la seconde partie du siècle, les évolutions urbaines semblent être beaucoup plus indépendantes de la présence de l'usine qu'auparavant, grâce à une importante diversification des activités économiques permise par le développement de zones d'activité et du centre hospitalier Lyon Sud, implanté en partie dans la commune.
En I97I, à un moment où le PCF apparaît peut-être moins radical qu'auparavant du fait de l'entente avec les socialistes et les radicaux de gauche autour du Programme Commun, l'élection de la liste PCF, à la faveur d'une triangulaire, renouvelle entièrement l'équipe municipale ${ }^{6}$. L'arrivée d'une équipe municipale PCF trouve peut-être plus profondément sa source dans des évolutions lentes, liées aux transformations économiques induites par l'industrie chimique depuis près de cinquante ans? .

\section{La défense du site industriel}

Lors de ses premières années d'activité, la municipalité a dû faire face à des pollutions importantes provoquées par des fuites d'acroléine, un gaz très toxique. Elle a porté plainte à plusieurs reprises contre le directeur de l'usine, aux côtés de la section $\mathrm{CGT}^{8}$. Par ailleurs, certaines décisions de la direction du site ont été interprétées par les élus communistes comme des signes de désengagement de la part du groupe industriel. En effet, la crainte de la désindustrialisation avait été avivée par un précédent : la fermeture en I978 du site Ugilor, filiale de Péchiney, site de production qui n'avait tourné que dix ans et fut jugé trop peu rentable au niveau européen. De nombreuses actions ont donc été menées par les élus pour contrer la menace de fermeture du site. Pour J.-M. Mick, maire de I97I à 200I, PUK procédait à «un chantage à l'emploi [sur le mode] : "si vous nous embêtez, on s'en va" ».

La municipalité affiche alors des positions industrialistes, appuyées sur l'idée promue par le MNLE9 que la lutte pour l'environnement ne devait pas être une lutte contre l'industrie mais contre ses logiques de profit, car le progrès technique serait à même de résoudre les problèmes de pollution. Selon le maire : «il y avait un problème d'économies [sur la sécurité] qui entraînait des pollutions régulières tant atmosphériques que du Rhône. Nous avons eu des pollu-

5. Dès I966, le groupe PCUK (Produits Chimiques Ugine Kuhlmann) qui deviendra par la suite PUK (Péchiney Ugine Kuhlmann), puis Atochem en I983, et en 2000 Atofina, filiale de Total-Fina-Elf.

6. Après un bref épisode de gestion communiste à la Libération, une équipe d'alliance entre trois courants républicains modérés, ex-SFIO, Union des Républicains et Mouvement Républicain Populaire, a dirigé la ville de I947 à I97I.

7. François Duchêne a noté un décalage similaire entre structuration économique et structuration politico-spatiale, dans le cas de l'agglomération de Roussillon (Isère), autre site chimique de la grande région lyonnaise. Cf. Duchêne F., (2002), Industrialisation et territoire. RhônePoulenc et la construction sociale de l'agglomération roussillonnaise, Paris, L'Harmattan, p. I74.

8. Pour l'histoire des pollutions provoquées par le site de PCUK entre I972 et I978, voir Guérin-Henni A., (I980), Les pollueurs. Luttes sociales et pollution industrielle, Éditions du Seuil, pp. 74-76.

9. Mouvement National de Lutte pour l'Environnement, association proche du PCF, fondée en I98I par le maire de Givors, un proche de J.-M. Mick. 
tions du Rhône absolument [terribles], et puis nous avions ce chantage à l'emploi !». Est ainsi soulignée l'insuffisance des mesures de sécurité, et non la dangerosité des productions ; mais les élus redoutent surtout que le site «disparaisse». Au début des années I990, alors que la municipalité est en bons termes avec le groupe Elf, qui a réalisé des améliorations évitant les rejets polluants, les Verts de l'agglomération lyonnaise vont faire entendre un nouveau type de contestation quant à la présence du groupe chimique. En effet, après l'interdiction des $\mathrm{CFC}^{\mathrm{I}}$, le groupe Atochem, qui en produisait à Pierre-Bénite, décide de fabriquer leurs substituts ${ }^{\text {II }}$ sur ce même site. Pour le maire, ceci est une garantie de pérennisation du site. Malgré les protestations des Verts, les procédures d'autorisation sont facilitées, permettant à l'industriel de démarrer rapidement la construction du nouvel atelier. Pour les responsables municipaux actuels, l'obtention de cette nouvelle production était dans l'intérêt de la ville, ce fut une «bataille» du maire autour d'un «enjeu international», car le groupe Atochem possédait d'autres sites à l'étranger.

Ces interventions en faveur du maintien de l'industrie chimique sont aujourd'hui présentées comme naturellement liées à la nature «ouvrière » de la commune, qualifiée de «ville de gauche». Car l'usine est érigée en élément fédérateur de la construction d'une identité consensuelle de la ville : comme l'explique G. Di Méo, «les acteurs sociaux, qui construisent et reconstruisent au quotidien le territoire, ne sélectionnent dans ce but que certaines séquences de son histoire. Ils retiennent celles qu'ils jugent les plus aptes à consolider l'identité socio-spatiale et à mobiliser les énergies locales, dans une perspective qui manque rarement d'arrières pensées politiques $\gg^{\mathrm{I2}}$. En I994, peu de temps après des actions de Greenpeace contre le site chimique, paraît dans le journal municipal un article intitulé «Ato Pierre-Bé : la ville et l'usine ${ }^{13}$. Reflétant un certain souci de légitimation de la politique municipale, cet article est révélateur de l'image publique que la municipalité souhaite donner de la commune, à travers la présentation d'une histoire partagée par l'établissement industriel et la ville. L'usine est d'abord présentée comme pourvoyeuse d'emplois par un syndicaliste CGT du site : «la population accepte le voisinage à risque avec Ato dans la mesure où l'usine procure des emplois». Puis l'on parle du rôle financier de l'industrie, porteur de bénéfices pour l'ensemble de la population : «Il est vrai que la commune trouve son compte, financièrement parlant, dans la présence d'Ato [...] par le biais de la taxe professionnelle. Un plus pour le budget communal qui permet la réalisation d'équipements dont profitent tous les Pierre-Bénitains. Cet élément contribue donc à rendre supportable une situation que les feux de l'actualité écologique et des accidents survenus ailleurs pourraient rendre plus inquiétante». L'accent est enfin

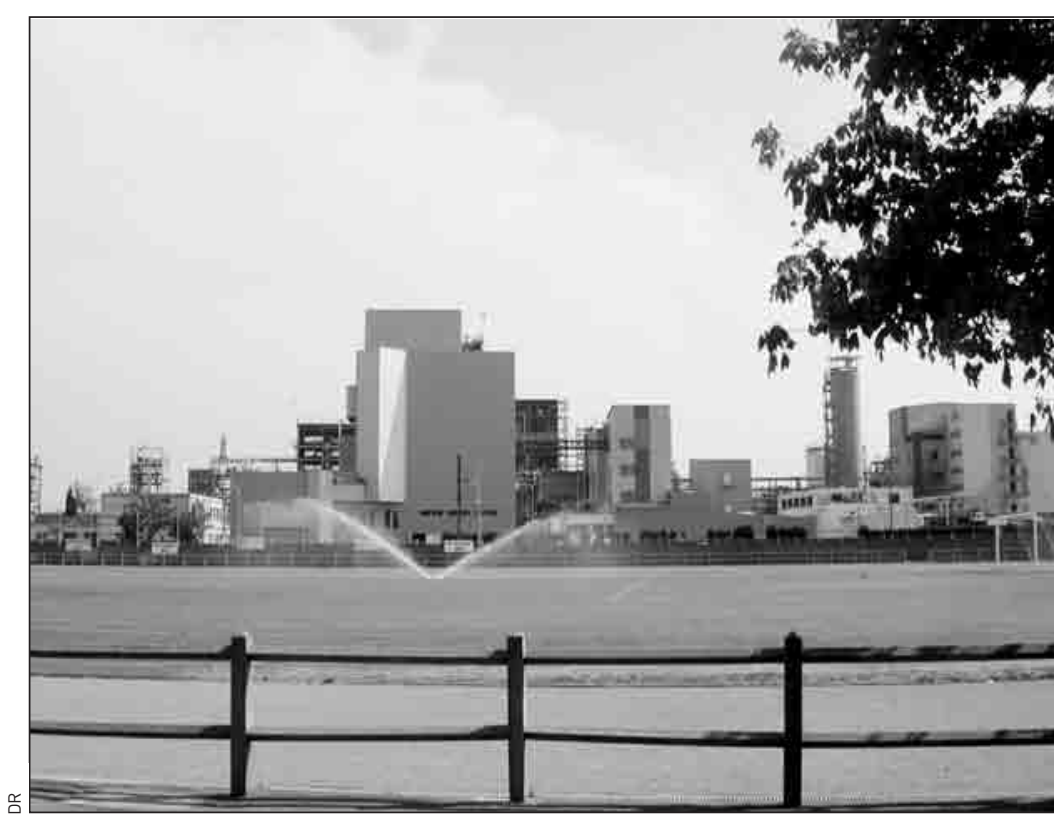

Le terrain de foot du stade de Pierre-Bénite et les ateliers de l'usine Atofina.

porté sur les liens établis de longue date entre les habitants et l'entreprise, l'histoire de l'immigration ouvrière étant mise en avant : «Les Pierre-Bénitains ont presque tous un père, un oncle, ou un cousin d'Ato». Ceci signale la nécessité pour les élus d'expliciter le sens de la présence industrielle chimique sur le territoire communal.

\section{Des enjeux économiques et identitaires}

La majorité municipale actuelle, à dominante communiste et affiliée à la «gauche plurielle» (mais ne comptant aucun élu issu des Verts), doit composer avec la mise en place de la législation sur les risques technologiques, qui prévoit l'information au public et contraint fortement les possibilités d'aménagement autour de l'établissement industriel. De plus, les élus reconnaissent que ni le nombre de salariés employés à l'usine, ni le montant de la taxe professionnelle revenant à la commune ne sont plus garantis à un niveau stable. En effet, les investissements sur le site ne se traduisent plus forcément par des embauches ni par le maintien d'un nombre élevé d'emplois. Enfin, la loi Chevènement du I2 juillet I999 prévoit, avec le pas-

Io. Les accords internationaux de Montréal en I987 ont considéré que ces produits étaient les principaux destructeurs de la couche d'ozone.

II. Les substituts utilisés actuellement sont les HFA, employés dans les systèmes de climatisation et de réfrigération.

I2. Di Méo G., (I998), Géographie sociale et territoires, Paris, Nathan université, p. 55 .

I3. Miroir journal municipal, avril I994. 
sage à la Taxe Professionnelle Unique (TPU), la répartition des ressources financières entre communes de l'agglomération. Les élus ont donc opéré une transition progressive dans leur évaluation des conséquences économiques et urbaines liées à la présence de l'usine.

Le retentissement de la catastrophe de Toulouse, accompagné de prises de position en faveur de la fermeture d'AZF, a donné lieu à la dénonciation par certains de l'existence de sites industriels à risque en milieu urbain. Mireille Elmalan, actuelle maire de la ville, appelée à réagir devant la menace de stigmatisation pesant sur Pierre-Bénite, s'est prononcée dans le débat national contre le départ des usines existantes. Elle a invité la direction du site Atofina à venir présenter en conseil municipal les mesures de sécurité, ce qui a donné lieu à un article intitulé «À l'unanimité : "Atofina doit rester" $\gg^{14}$, paru dans le journal municipal. Or un certain nombre d'élus travaillent sur le site ou dans le secteur de la chimie, et l'on peut sans peine envisager la difficulté qu'il y a pour les membres du Conseil municipal, eux-mêmes résidant à proximité plus ou moins grande de l'usine, à évoquer frontalement sa dangerosité. Comme l'a montré F. Zonabend à propos des riverains et des travailleurs de l'usine nucléaire de la Hague, «pour vivre dans des conditions de confort moral suffisantes, il ne faut pas que l'on se rappelle ou que l'on vous rappelle constamment que vous habitez une zone spéciale et allez travailler dans un établissement dangereux $»^{15}$.

Pour qu'une telle situation reste supportable, ceux qui la vivent se trouvent devant la nécessité de lui donner un sens. La maire actuelle explique en mai 2002 que l'usine de Pierre-Bénite «fait partie de la ville», et qu'il est difficile d'envisager l'avenir sans sa présence : « s'il n'y a plus [d'usine], Pierre-Bénite va être une ville dortoir, c'est-à-dire une ville qui meurt. Il n'y aura plus de centre-ville, plus d'animation. [...] Car l'usine [...] c'est aussi des échanges entre clubs sportifs de l'usine et de la ville, des échanges au niveau culturel avec le comité d'entreprise. Cela fait vivre la ville, pas seulement sur le plan financier, mais également sur le plan de l'animation ». Cette évocation n'est pas sans rappeler la menace de la désindustrialisation ressentie dans un contexte différent par les municipalités communistes précédentes. La maire mobilise également l'«attachement des Pierre-Bénitains à leur usine», afin d'ancrer son engagement politique dans une histoire longue et un contexte social qu'elle estime favorable au maintien de l'industrie chimique.

\section{Aménagement et inégalités socio-spatiales}

La première action des élus dans le domaine de l'aménagement urbain après I97I a consisté à bloquer l'implantation de i 800 nouveaux logements, initialement prévue par un projet de I966, au motif de désé- quilibres induits par la construction rapide de près de 600 logements à Haute-Roche. Ce n'est qu'en I98I que les élus, affirmant «le droit au logement de qualité pour tous », lancent une ZAC «à vocation », entièrement maîtrisée par la commune grâce aux revenus de la taxe professionnelle. Pour les villes à municipalités communistes en place dans les années I960, I970 ou I980, l'intervention en faveur du logement, dans un souci de progrès social, visait à résorber l'exclusion des classes populaires par l'accès à un habitat de qualité ${ }^{16}$. À Pierre-Bénite, en cohérence avec les luttes pour la sauvegarde de l'emploi ouvrier sur le site chimique, le projet de ZAC, orienté vers l'accession à la propriété des «ouvriers qualifiés », selon les termes du maire, intégrait un effort pour unir la population des quartiers de la commune. Il devait être le levier d'une restructuration complète du centre-ville.

Mais ces projets étaient également sous-tendus par la nécessité de résoudre des problèmes d'ordre social, prégnants mais peu explicités. La commune a connu entre 1975 et 1982 une baisse de sa population ${ }^{17}$ ainsi qu'une certaine désaffection du centre ancien. Au sujet de cette période, les propos de l'ancien chargé de mission du maire oscillent entre la désignation pudique de la situation de précarité des habitants du centre ancien - personnes âgées ayant peu de ressources, familles immigrées occupant un habitat peu salubre, personnes sans emploi - et le constat de la dégradation du bâti. La municipalité a mobilisé dès les années i980 d'importants moyens financiers pour mener une politique d'achat dans cette zone et pallier les effets de la paupérisation et de la dégradation redoutées en louant des appartements réhabilités. Ces interventions étaient par ailleurs justifiées par l'utilité des réserves foncières pour la future opération de «reconquête» du centre.

Le diagnostic urbain de I992 met en avant l'existence d'«un puzzle de territoires aux caractéristiques singulières $»^{18}$. Or la critique des dysfonctionnements urbains induits par l'hétérogénéité morphologique de la ville renvoie de fait à des clivages sociaux, bien visibles si l'on regarde la répartition des CSP dans la population des différents quartiers. Pierre-Bénite a en effet été sujette à des processus complexes de fragmentation socio-spatiale, liés à l'histoire de la constitution des

I4. Miroir journal municipal, $\mathrm{n}^{\circ} 86$, novembre 200I, p. 3 . I5. Zonabend F., (I989), La Presqu'île au nucléaire, Paris, Éditions Odile Jacob, p. I2

I6. Cf. Althabe G., Légé B., Sélim M., (1984), Urbanisme et réhabilitation symbolique Ivry. Bologne. Amiens, Paris, Anthropos.

I7. La commune est passée d'un peu plus de ro ০০o habitants en I975, à 9500 environ en I982, pour en compter 9900 environ en I999.

I8. Plan de référence de Pierre-Bénite : diagnostic phase I, Communauté urbaine de Lyon, Cité Plus, février I992. 
quartiers, à leurs situations respectives au sein de la commune, ainsi qu'à des changements sociaux plus globaux $^{19}$. Le Perron, quartier issu de la ZAC, constitue finalement avec le «haut» de la ville un ensemble pavillonnaire abritant des classes moyennes/aisées. L'offre de logements privatifs y a induit une sélection de la population, contribuant à accentuer les disparités avec le centre ancien et Haute-Roche, beaucoup plus proches de l'usine chimique et de l'autoroute ( $c f$. la carte urbanisation et infrastructures).

\section{La négociation des zones de risques}

La maîtrise de l'urbanisation est intervenue en I990 dans ce contexte local porteur d'enjeux multiples et interdépendants, en termes de constitution sociospatiale de la trame urbaine. Emmanuel Martinais a ainsi montré, à propos de la commune de SaintFons $^{2 \circ}$, que les considérations des élus, relativement à la gestion urbaine locale, ont conduit à une modification des périmètres issus des études de danger de la DRIRE ${ }^{2 I}$, en vue de leur affichage dans le POS. À Pierre-Bénite, cette procédure a fait l'objet d'une négociation directe entre le maire, le Préfet et la DRIRE. La zone $\mathrm{Z}_{2}{ }^{22}$ retenue épouse dans son tracé les limites des parcelles de petite taille du centre ancien, mais traverse en arc de cercle les parcelles de Haute-Roche, d'étendue beaucoup plus vaste (cf. la carte des zones $Z_{I}$ et Z2). L'ancien maire nous a confirmé qu'il avait pu intégrer ses préoccupations concernant le centre ancien dans la négociation, alors que le quartier d'habitat collectif de Haute-Roche ne nécessitait pas une attention particulière, étant donné l'impossibilité d'y mener des opérations nouvelles.

Mais une fois ce tracé négocié et inscrit dans le POS, aucune modification, même légère, n'a pu être obtenue par le nouveau maire, qui souhaitait régler un litige dû au cas d'une parcelle coupée par la zone. Par ailleurs, il semble que les responsables municipaux n'aient pas eu connaissance de la genèse du découpage de la zone $\mathrm{Z}_{2}$, et leurs explications à ce sujet sont aujourd'hui très vagues. Comme l'a montré E. Martinais, «à mesure que la mémoire des acteurs locaux s'estompe (ou qu'ils disparaissent de la sphère locale), l'assimilation s'opère entre d'une part, ce qui n'est que le produit d'une négociation sociale (par exemple une cartographie des risques) et d'autre part un fait technique "pur" marqué du sceau de la scientificité»23. Si une solidification du tracé des zones s'est opérée, de nouvelles négociations ont eu lieu au sujet de l'application des restrictions de constructibilité 44 à l'intérieur de la zone $Z_{2}$, du fait de nécessités locales en matière d'équipements publics, pour la plupart présents dans le centre et donc inclus dans la zone Z2. L'inspecteur de la DRIRE pour le site de Pierre-Bénite reconnaît d'ailleurs que l'affirmation par les services de l'État d'un principe de non remise en cause de l'existant oblige à reconsi-

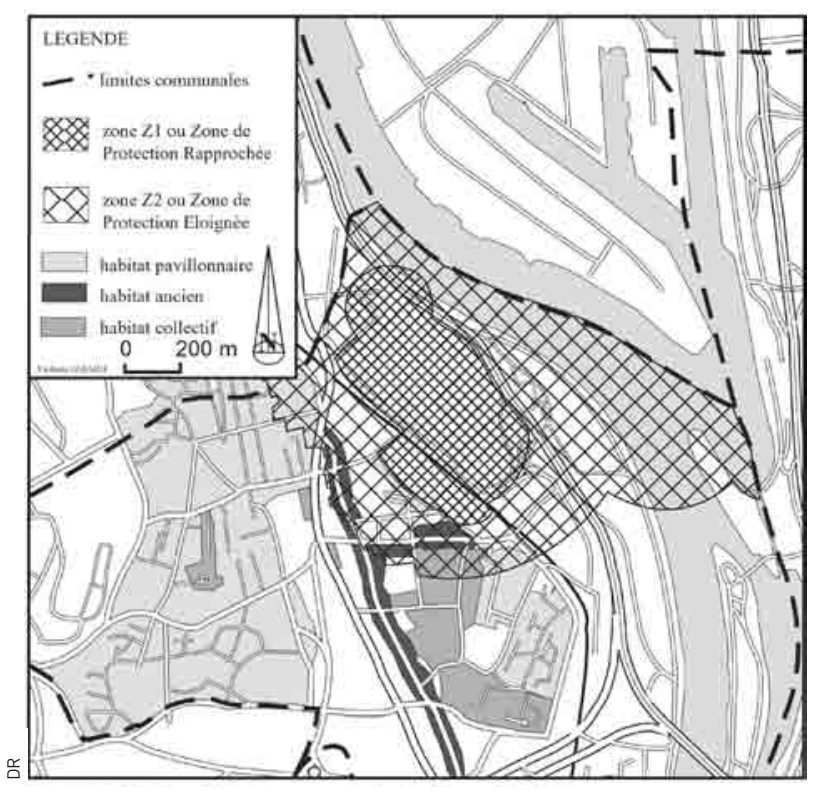

Les zones Z1 et Z2.

dérer et à réinterpréter la réglementation pour arriver à un compromis avec les élus locaux concernant les équipements et les constructions déjà en fonction. En outre, les moyens financiers et fonciers de la commune ont permis de résoudre les problèmes liés au manque d'espace dans les locaux publics.

La gestion urbaine du risque n'a donc pas changé la définition des représentations des élus à l'égard des différents quartiers de la commune. Au contraire, la présence de la zone $\mathrm{Z}_{2}$ dans le centre ancien et Haute-

I9. Sur ce point, cf. Bacqué M.-H., Fol S., (1997), Le devenir des banlieues rouges, Paris, L'Harmattan, p. 202.

20. Martinais E., (200I), Les sociétés locales à lépreuve du risque urbain. Un siècle de gestion du danger dans deux contextes de l'agglomération lyonnaise, thèse de géographie/aménagement, dir. A. Vant, Université Jean Monnet de Saint-Étienne.

2I. Direction régionale de l'industrie, la recherche et l'environnement. 22. Il existe deux types de zones, $Z_{I}$ et $Z_{2}$ dans lesquelles s'appliquent respectivement l'interdiction et les restrictions de constructibilité.

23. In Coanus T., Duchêne F., Martinais E., (2000), La ville inquiète. Développement urbain, gestion du danger et vie quotidienne sur trois sites «à risque» de la région lyonnaise (fin XIX ${ }^{e}-$ fin XXe siècle), rapport financé par le contrat de plan État/Région Rhône-Alpes, Laboratoire RIVES, p. 336 .

24. La réglementation de la zone $\mathrm{Z}_{2}$ permet les constructions nouvelles de COS inférieur à o,15, ce qui dans les faits est impossible à Pierre-Bénite, au vu de la densité existante. Le rapport parlementaire du 29 janvier 2002 sur la sûreté des installations industrielles relève effectivement que l'application des instruments de maitrise de l'urbanisation «conduit à geler d'une manière radicale toute construction dans les zones exposées au risque» (in Loos F. (Président), Le Déaut J.-Y. (rapporteur), (2002), Rapport sur la sûreté des installations industrielles et des centres de recherche et sur la protection des personnes et de l'environnement en cas d'accident industriel majeur, rapport $n^{\circ} 3559 \mathrm{du}$ 29/0I/2002, tome I, Assemblée Nationale, p. II5. 
Roche a renforcé la nécessité d'y intervenir pour en éviter la relégation complète, tout en restreignant les moyens d'action disponibles. La municipalité a alors mené de multiples actions sectorielles de réhabilitation ${ }^{25}$ et de valorisation des espaces publics, permises par la législation.

\section{Incertitude pour la revalorisation du centre-ville}

En matière de politique du logement, le Plan Local de l'Habitat (I998) prévoit de «satisfaire le maintien sur place d'habitants ne trouvant pas de bonnes conditions de logement, mais aussi d'attirer de nouvelles populations susceptibles de rééquilibrer et redynamiser la composition socio-démographique du centre». Il s'agit d'un positionnement nouveau des élus, en faveur d'un aménagement du centre ancien orienté vers la satisfaction d'exigences propres aux classes moyennes ou supérieures, en termes de qualité de vie et de logement. Ce changement signale que les élus souhaitent mettre fin à une certaine spécificité ouvrière ${ }^{26}$, dans la définition de l'identité et surtout de l'image de la commune, notamment vis-à-vis des communes voisines, généralement qualifiées dans l'agglomération lyonnaise de «résidentielles» et apparaissant plus attractives pour des classes sociales favorisées ${ }^{27}$. La chute du nombre d'emplois dans le site chimique, la montée tendancielle du chômage dans les années I980, un certain vieillissement du centre, les évolutions du quartier d'habitat social considérées comme problématiques, sont autant de considérations, non exposées explicitement, qui ont pu infléchir la politique municipale, tandis que la composition des Conseils municipaux et les cadres idéologiques du PCF ont également évolué. Ceci suscite toutefois certains questionnements au sein de la majorité municipale quant au devenir de son électorat; d'autre part, les élus restent muets sur le fait que la présence de l'usine, source de danger et de nuisances, puisse induire une certaine dépréciation du cadre communal pour des ménages aisés. Si Pierre-Bénite n'est pas à proprement parler une ancienne «banlieue rouge», on peut cependant penser que les politiques locales évoluent à mesure que les modalités de peuplement se transforment ${ }^{28}$.

L’ancien maire a lancé en I999 le projet de ZAC du centre, un «programme de logements diversifiés» offrant une alternative au parc ancien. C'est l'occasion pour les élus communistes d'affirmer leur effort de revalorisation de la ville, sur le plan du cadre urbain comme de la représentation sociale qui s'en dégage. Cette opération est contiguë à la zone Z2, le premier magistrat de la commune ayant déjà eu en tête ce projet de ZAC lors de la négociation du tracé de la zone $Z_{2}$ en I990. Concernant le quartier Haute-Roche, objet d'une procédure Contrat de Ville, la maire actuelle souhaiterait recourir à des démolitions/ reconstructions selon l'esprit de la loi SRU, afin de rétablir une mixité sociale. Ainsi, la municipalité s'appuie sur les espaces extérieurs à la zone $\mathrm{Z}_{2}$ pour impulser un développement bénéfique à l'ensemble du centre. Pour autant, les techniciens et les élus soulignent que la ZAC n'est qu'un premier pas vers la restructuration. Ils se trouvent devant deux constats inconciliables : d'une part un travail de grande ampleur reste à accomplir pour aboutir à un centre « sauvé », selon l'expression de l'urbaniste territorial de la Communauté urbaine de Lyon; et d'autre part la présence de la zone Z2 implique l'impossibilité d'y entreprendre des actions importantes.

À la suite de la catastrophe de Toulouse, et alors que la TPU va priver la municipalité de ressources propres assez élevées, la maire, M. Elmalan, redoute un durcissement de la législation, et dit même avoir eu peur que cet accident ne vienne interrompre la réalisation de la ZAC du centre. De surcroît, la thématique du risque est de plus en plus présente à l'esprit de services de l'État autres que la DRIRE (DDE, Sécurité Civile), tandis que pour la Communauté urbaine, le «Couloir de la Chimie» est une préoccupation importante. Le maire exprime donc ses craintes pour le devenir de la commune, pour elle entièrement soumis aux évolutions de la législation, et redoute son impuissance en cas de mesures plus strictes : «S'ils accentuent les périmètres autour des usines, on ne va pourtant pas pouvoir transporter Pierre-Bénite à Millery ou à Charly 29 ! Or on n'a pas d'espaces, donc on se demande comment faire. Aujourd'hui, dans la zone de vigilance d'Atofina, on a la Maison du Peuple ${ }^{30}$, les

25. Une OPAH communautaire a notamment été menée de 1993 à I995 que la municipalité a ensuite poursuivie par une Animation communale d'amélioration de l'habitat.

26. En I990 et I999 la catégorie des ouvriers prédomine dans la commune, représentant 30 et $28 \%$ de la population selon les recensements INSEE, la proportion relative avec les professions intermédiaires et les cadres étant inversée par rapport aux moyennes de l'agglomération lyonnaise.

27. Depuis le début des années quatre-vingt, les travaux du groupement d'urbanisme du sud-ouest de la Communauté urbaine de Lyon signalent la particularité de Pierre-Bénite, commune presque entièrement urbanisée et accueillant des sites industriels, apparaissant au côté d'Oullins comme une «ville», par contraste avec les communes voisines du sud ou de l'ouest (Saint-Genis-Laval, Irigny, Charly, Vernaison) abritant presque exclusivement des zones pavillonnaires.

28. Ainsi Marie-Hélène Bacqué et Sylvie Fol soulignent à propos de la ville de Saint-Denis (93) que «les évolutions des politiques locales et des discours municipaux se comprennent comme des adaptations à la réalité sociale des villes [...] et traduisent également la transformation progressive de l'image sociale de ces communes de banlieue, tiraillées entre un processus de paupérisation, et un autre, inverse, de diversification sociale "par le haut" » (op. cit., p. I03).

29. Millery et Charly sont deux communes résidentielles de l'ouest lyonnais.

30. La Maison du peuple est la salle de spectacles et le centre culturel de Pierre-Bénite. 
écoles, la Poste, la mairie, le stade, le marché ! [...] C'est une ville qui va mourir...». L'usage métaphorique de la «mort de la ville», complétant l'affirmation selon laquelle l'usine la fait «vivre», est certainement lié aux représentations communément partagées de territoires en proie à une désindustrialisation massive. Mais elle nous paraît également résonner avec le danger d'un accident technologique - danger qui n'est jamais évoqué ouvertement, même si indirectement la législation sur les risques le rappelle constamment lourd de significations symboliques, qui trouvent peutêtre à s'exprimer par ce biais là. Les responsables techniques de l'urbanisme parlent également de la ville en des termes fortement dépréciatifs, empreints d'inquiétude pour l'avenir : la commune serait «bloquée » par les contraintes, «étouffée» entre le manque de terrains disponibles et les interdictions de construire à l'intérieur des périmètres.

\section{La mise en regard du risque et du développement}

Plusieurs types de menaces pour la ville ont été identifiés depuis trente ans par les élus locaux ; le risque technologique est loin d'être (et d'avoir été) leur seule source de préoccupation. Les élus se sont notamment engagés à plusieurs reprises contre le départ de l'usine, par crainte de la désindustrialisation et des pertes d'emploi. Ils ont construit pour cela un système de représentations permettant d'envisager la présence du site industriel de façon cohérente, combinant histoire locale, identité ouvrière et rôle économique. La façon d'envisager le risque technologique, ainsi que la place à lui accorder dans les actions municipales, ont donc été perpétuellement construites et adaptées en fonction notamment de difficultés économiques rencontrées par la population locale, des orientations politiques du PCF, des ressources financières et de l'apparition, sur le plan national et international, d'un espace social et politique d'investissement des questions environnementales et de sécurité.

Relativement à la gestion du risque, la maîtrise de l'urbanisation a été négociée de façon différenciée selon les composantes socio-spatiales des différents quartiers de la ville, et donc sur la base d'enjeux territoriaux déterminés par la municipalité. Les réponses sociales apportées au risque sont donc le résultat de processus d'élaboration s'effectuant pour une bonne part localement, en fonction de la situation économique, sociale et politique du territoire. Dès lors, on peut réinterroger différemment le «paradigme du risque», supposé se diffuser de façon uniforme, continue et nécessaire dans une société qui changerait ainsi

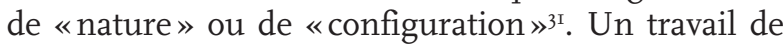
terrain, attentif à la profondeur historique des relations ville/industrie comme aux dynamiques sociospatiales, révélerait plutôt l'existence d'une forte spécificité du territoire local. Contrairement à l'hypothèse de l'avènement d'une "société du risque», dans laquelle l'ensemble des relations sociales seraient vues à travers ce prisme, du point de vue des acteurs locaux, la question du risque est toujours associée aux autres enjeux sociaux de développement.

Violaine Girard

3I. Beck U., (200I), La société du risque. Sur la voie d'une autre modernité, Paris, Aubier, p. 38.

Violaine Girard est ingénieur des Travaux Publics de l'État, doctorante en anthropologie sociale et ethnologie - Laboratoire RIVES, ENTPE (groupe thématique « Risques urbains »).

$<$ GIRARD_V@entpe.fr > 